\title{
$\mathbf{F e}-\mathrm{Ni}-\mathrm{Si}-\mathrm{B}$ 非晶合金中的原子扩散
}

\author{
姜洪刚 丁炳哲 王景唐 胡壮蜞 \\ (中国科学院金属研究所, 沈阳 110015)
}

\section{关链词非晶合金、原子扩散、表面偶析}

\section{1 实 验}

非晶合金 $\mathrm{Fe}-\mathrm{Ni}-\mathrm{Si}-\mathrm{B}$ 条带由单辊激冷法制备. 选取条带的亮面抛光,得到光滑的表面， 再将它放到丙酮液中超声清洗, 用酒精冲洗吹干, 样品在真空室中被固定在由电阻丝加热的铜 块上,温度测量采用铜-康铜热电偶.

原位退火过程中元素的表面偏析随退火时间变化的测量在 LAS-3000 RIBER 俄歇 (A ES) 表面分析系统中进行, 选取适当的时间间隔测定各元素的峰高值 (APPH), 退 火温 度范围为 $673-753 \mathrm{~K}$, 系统真空度为 $10^{-7} \mathrm{~Pa}$.

先对抛光过的样品表面进行 Ar 离子轰击, 去掉表面玷污层, Ar 电压为 $2.5 \mathrm{kV}$. 将清洗 过的样品加热到 $573 \mathrm{~K}$, 此时氧偏析很严重, $\mathrm{Si}$ 向自由表面的偏析被抑制. 为了清除氧偏析对 $\mathrm{Si}$ 偏析的影响, 在保持 $\mathrm{Ar}^{+}$束流轰击的条件下,将样品在 373 - $573 \mathrm{~K}$ 之间进行 5 个周期的加热 冷却处理, 直到氧信号近乎消失, $\mathrm{Si}$ 向自由表面的偏析变得很明显, 然后将样品加热到 $673 \mathrm{~K}$, 待温度平衡后, 停掉 $\mathrm{Ar}^{+}$束流, 立即记录元素俄歇峰随退火时间的变化, 实验的温度依次升高 到 $693,713,733$ 和 $753 \mathrm{~K}$.

\section{2 结果与讨论}

Hofmann 和 Erlewein 研究了原子向自由表面偏析的动力学过程, 给出了偏析原子的表 面浓度 $\left(C_{\mathrm{s}}\right)$ 随退火时间 $t$ 的变化关系为 ${ }^{[1]}$

$$
C_{1}=C_{0}\left\{1+2 / a(D t / \pi)^{1 / 2}\right\},
$$

其中 $C_{0}$ 为偏析原子的体浓度, $a$ 为单夽偏析膜厚度, $D$ 为偏析原子的体扩散系数. 因为元素的 峰高值 (APPH) 与其表面浓度 $C$ 。成正比, 故对选定的 $\mathrm{Si}$ 元素, 方程 (1) 可写成

$$
\mathrm{APPH}=2 k\left(C_{0} / a\right)(D t / \pi)^{1 / 2},
$$

其中 $k$ 为常数. 曲线 $\mathrm{APPH}-t^{1 / 2}$ 的斜率为

$$
G=2 k C_{0} D^{1 / 2} / a \pi^{1 / 2},
$$

其中 $G$ 为偏析曲线的初始段斜率, 对符合 Arrhenius 关系的扩散 $D-D_{0} \exp (-E / R T)$, 将 此式代入(3)式中可得

$$
\ln G=\ln A-E / 2 R T \text {. }
$$


从式中可以明显看出, $\mathrm{Si}$ 原子的扩散激活能可由 $\ln G-1 / T$ 曲线的斜率求得.

正式偏析实验前在 373-573K 之间的周期退火, 一方面可以促进氧原子向自由表面偏 析, 然后被 Ar 离子剥离掉, 直到最后氧信号变得很微弱, $\mathrm{Si}$ 向自我表面偏析变得明显; 另一方 面可以使亚稳态非晶合金趋向于标准驰豫态, 从而使表面偏析更接近平衡过程.

原位退火温度 (673-753K) 的选定基于以下考虑:

1) 保证有明显的 Si 向自由表面偏析.

2) 去除氧偏析对 Si 偏析的影响.

3) 避免非晶合金发生晶化, 对所研究的非晶 $\mathrm{Fe}_{73} \mathrm{Ni}_{5} \mathrm{Si}_{12} \mathrm{~B}_{10}$ 和 $\mathrm{Fe}_{63} \mathrm{Ni}_{10} \mathrm{Si}_{12} \mathrm{~B}_{10}$ 合金, 用差热扫描分析 (DSC), 在 $20 \mathrm{~K} / \mathrm{min}$ 的加热速率下,测得的晶化温度分别为 815 和 $800 \mathrm{~K}$. 图 1 为不同温度下,原位退火过程中 Si $(92 \mathrm{eV})$ 俄歇峰高随退火时间开方的变化,曲线初始阶 段的线性关系表明,公式(1)可被用来求 $\mathrm{Si}$ 的扩散系数,图 2 表示典型的 $\mathrm{Si}$ 扩散的 Arrhenius
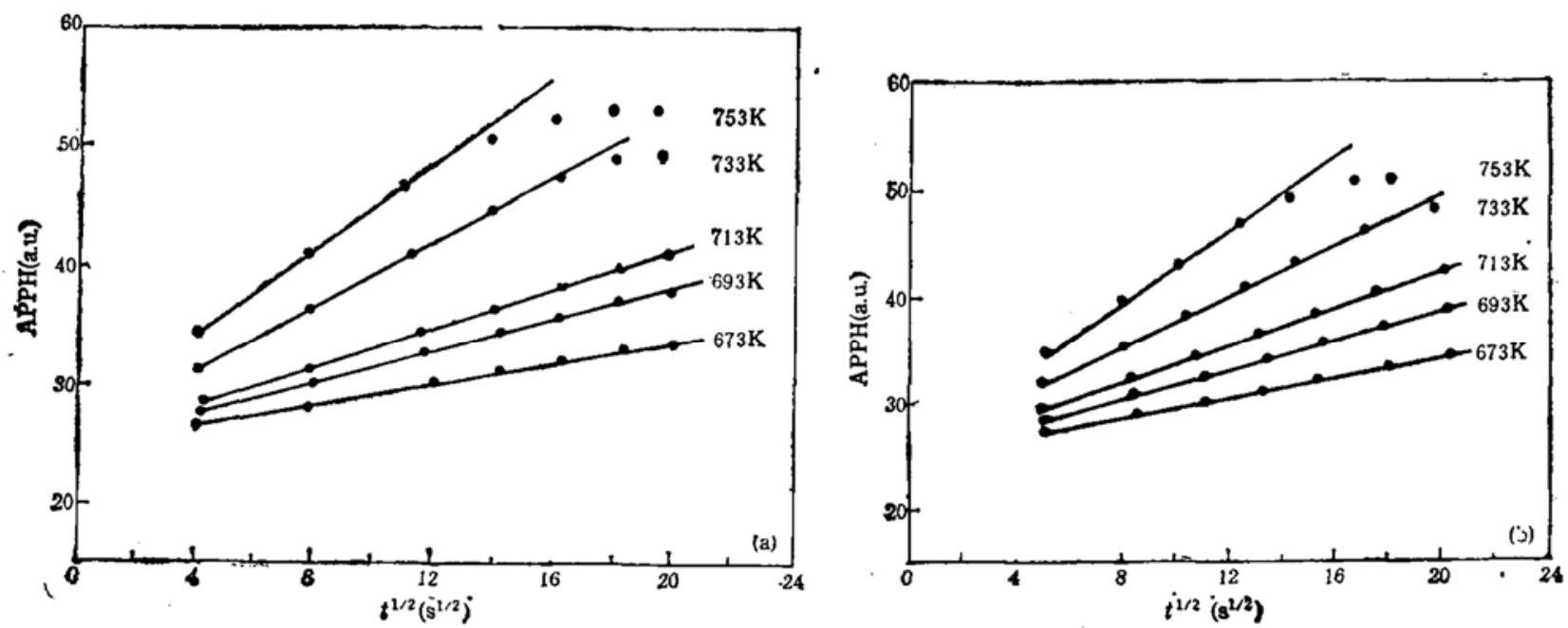

图 1 不同温度下,原位退火过程中 $\mathrm{Si}(92 \mathrm{eV})$ 俄歇峰高随退火时间开方变化曲线

(a) $\mathrm{Si}$ in $\mathrm{Fe}_{3} \mathrm{Ni}_{1} \mathrm{Si}_{12} \mathrm{~B}_{10}$, (b) $\mathrm{Si}$ in $\mathrm{Fe}_{6}, \mathrm{Ni}_{10} \mathrm{Si}_{12} \mathrm{~B}_{10}$

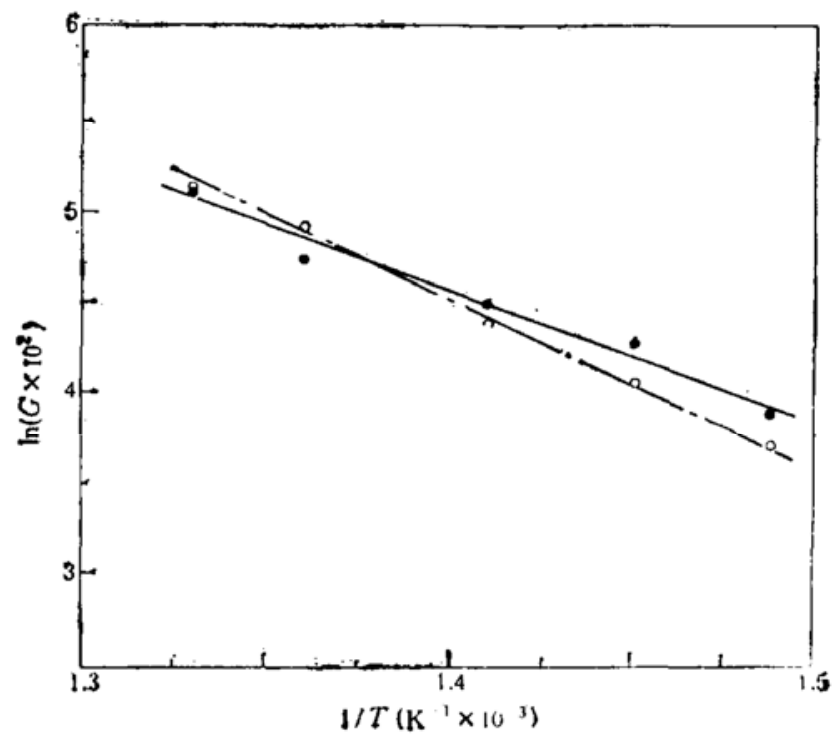

洛 2 Si 扩散的 Arrhenius 关系特征

$\bigcirc$ 为 $\mathrm{Fe}_{13} \mathrm{Ni}_{5} \mathrm{Si}_{12} \mathrm{~B}_{10},-$ 为 $\mathrm{Fe}_{68} \mathrm{Ni}_{10} \mathrm{Si}_{12} \mathrm{~B}_{10}$ 。 
关系特征，从其斜率可以求得 $\mathrm{Si}$ 在非晶合金 $\mathrm{Fe}_{73} \mathrm{Ni}_{5} \mathrm{Si}_{12} \mathrm{~B}_{10}$ 和 $\mathrm{Fe}_{69} \mathrm{Ni}_{10} \mathrm{Si}_{12} \mathrm{~B}_{10}$ 中的扩散激活 能分别为 149.6 和 $116.3 \mathrm{~kJ} / \mathrm{mol}$.

根据公式(3)和(4)可以求得 $\mathrm{Si}$ 在非晶合金 $\mathrm{Fe}-\mathrm{Ni}-\mathrm{Si}-\mathrm{B}$ 中的扩散系数,公式中常数 $k$ 由 Davis 等提供的方法 ${ }^{[2]}$ 求得, $\mathrm{Si}$ 的体浓度为 $C_{0}=12 \mathrm{at} \%$. Si 扩散的 Arrhenius 曲线见图 3, 其数学表达式为 $D=D_{0} \exp (-E / R T)$, 其中对 $\mathrm{Fe}_{73} \mathrm{Ni}_{5} \mathrm{Si}_{12} \mathrm{~B}_{10}, D_{0}=5.4 \times 10^{-8} \mathrm{~m}^{2} \mathrm{~s}^{-1}, E=$ $149.6 \mathrm{~kJ} / \mathrm{mol}$, 对 $\mathrm{Fe}_{68} \mathrm{Ni}_{10} \mathrm{Si}_{12} \mathrm{~B}_{10}, D_{0}=2.6 \times 10^{-10} \mathrm{~m}^{2} \mathrm{~s}^{-1}, E=116.3 \mathrm{~kJ} / \mathrm{mol}$, 可以看出, $5 \mathrm{a} \% \mathrm{Ni}$ 加入置换 $5 \mathrm{at} \% \mathrm{Fe}$, 非晶 $\mathrm{Fe}_{23} \mathrm{Ni}_{5} \mathrm{Si}_{12} \mathrm{~B}_{10}$ 合金扩散的频率因子和扩散激活能都有所 降低。

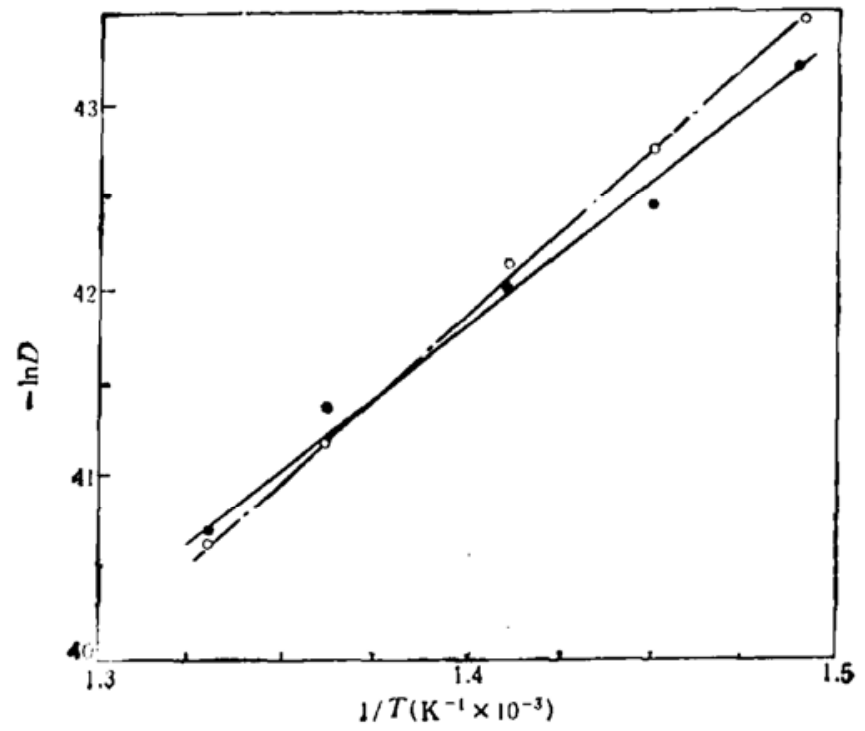

图 $3 \mathrm{Si}$ 扩散的 Arrbenius 曲线

○为 $\mathrm{Fe},{ }_{3} \mathrm{Ni}_{3} \mathrm{Si}_{12} \mathrm{~B}_{10},-$ 为 $\mathrm{Fe}_{60} \mathrm{Ni}_{10} \mathrm{Si}_{13} \mathrm{~B}_{10}$

表 $1 \mathrm{Si}$ 在非畨合金中的扩散系数

\begin{tabular}{|c|c|c|c|c|c|}
\hline $\begin{array}{l}\text { 合 金 } \\
(\mathrm{at} \%)\end{array}$ & $\begin{array}{c}\text { 扩散系数因子 } \\
\left(\mathrm{m}^{2} \mathrm{~s}^{-1}\right)\end{array}$ & $\begin{array}{c}\text { 激活能 } \\
(\mathrm{kJ} / \mathrm{mol})\end{array}$ & $\begin{array}{l}\text { 温度 } \\
(\mathrm{K})\end{array}$ & $\mid \begin{array}{c}\text { 扩散系数 }(650000) \\
\left(\mathrm{m}^{2} \mathrm{~s}^{-1}\right)\end{array}$ & 参考文献 \\
\hline $\mathrm{a}-\mathrm{Fe}_{\mathrm{e}_{2} \mathrm{~B}_{18}}$ & $5.7 \times 10^{-1}$ & 193 & $570-640$ & $1.8 \times 10^{-11}$ & [3] \\
\hline $\mathrm{a}-\mathrm{Fe}_{21} \mathrm{~B}_{13 .}, \mathrm{Si}_{3.5} \mathrm{C}_{2}$ & $11.7 \times 10^{-15}$ & 72 & $620-720$ & $2.8 \times 10^{-21}$ & {$[4]$} \\
\hline $\mathrm{a}-\mathrm{Fe}{ }_{3} \mathrm{Ni}_{3} \mathrm{Si}_{12} \mathrm{~B}_{10}$ & $15.4 \times 10^{-8}$ & 149.6 & $673-753$ & $4.7 \times 10^{-20}$ & \\
\hline $\mathrm{a}-\mathrm{Fe}_{63} \mathrm{Ni}_{10} \mathrm{Si}_{12} \mathrm{~B}_{10}$ & $12.6 \times 10^{-10}$ & 116 & $673-753$ & $7.0 \times 10^{-20}$ & \\
\hline
\end{tabular}

表 1 中列出了实验结果与文献 $[3,4]$ 中结果的比较. 显然, 实验所测得的扩散数据均在合 理范围内, 但与类似的系统中的扩散数据有一定的差别. 这是由于不同成分的扩散基体内部原 子周围的化学环境不同, 并且 Si 原子与基体元素之间的交互作用随合金元素的加人而改变, 所以扩散参数必然有差别. 另一方面, 非晶合金本质上处于结构亚稳态, 其自身结构在扩散退 火过程中趋向于标准驰豫态, 这样扩散系数就会受到影响, 所以非晶合金中扩散系数的比较是 十分困难的. Cantor 等人国叙述了弛豫对非晶合金扩散系数的影响,但未能得到统一的结果, 因不同的实验所得出的结论各不相同, 究其原因可能是非晶合金样品的制备以及扩散测量方 法不同所致。 
非晶合金具有复杂的结构, 目前还没有找到固定的模型来成功地描述, 通常在晶体中的缺 降一空位和间隙在非晶合金中极不稳定, 因而非晶合金中不存在严格的空位和间隙扩散. 研究 认为, 非晶合金中原子的扩散主要通过多个原子的协作式运动进行, 特别是当指数前因子 $D_{0}$ 特别大或特别小时.

\section{3 结 论}

俄歇 (AE.S) 原位表面偏析被成功地用来研究 $\mathrm{Si}$ 原子在 $\mathrm{Fe}-\mathrm{Ni}-\mathrm{Si}-\mathrm{B}$ 非晶合金中的原子 扩散,实验结果表明,在673-753K 温区内, $\mathrm{Si}$ 原子扩散符合 Arrhenius 关系,对 $\mathrm{Fe}_{73} \mathrm{Ni}_{5} \mathrm{Si}_{12}-$ $\mathrm{B}_{10}$ 非晶合金, 其频率因子 $D_{0}=5.4 \times 10^{-8} \mathrm{~m}^{2} \mathrm{~s}^{-1}$, 扩散激活能 $E=149.6 \mathrm{~kJ} / \mathrm{mol}$, 而对 $\mathrm{Fe}_{68}-$ $\mathrm{Ni}_{10} \mathrm{Si}_{12} \mathrm{~B}_{10}$ 非晶合金 $D_{0}=2.6 \times 10^{-10} \mathrm{~m}^{2} \mathrm{~s}^{-1}, E=116.3 \mathrm{~kJ} / \mathrm{mol}$.

\section{参孝文献}

(1) Hof mann, S., Erlewein, J., Surface Science, 1978, 77: 591-602.

(2) Davis, L.E., McDonald, N.C., Palmberg, P.W. et al. (eds.), Handbook of Auger Electron Spectro. scopy, 2nd ed., Physical Electronic, Divison, Perkin-Elmer, Eden Prairie, MN, 1978.

(3) Luborsky, F.E., J. Appl. Phys., 1983, 54 (10): 5732-5737.

(4] Vanwyk, G.N., Roos, W. D., Appl. Surf. Sci., 1986, 26: 317-325.

[5] Cantor, B., Cahn, R. W., in Amorphous Metallic Alloys (ed. Luborsky, F. E.), Butterworths, London, 1983, 487-505. 